\title{
Select-divide-and-conquer method for large-scale configuration interaction
}

\author{
Carlos F. Bunge ${ }^{a)}$ \\ Instituto de Física, Universidad Nacional Autónoma de México, Apdo. Postal 20-364, México 01000, \\ México \\ Ramon Carbó-Dorca ${ }^{\text {b) }}$ \\ Instituto de Química Computacional, Universitat de Girona, Campus de Montilivi, 17071 Girona, Spain
}

(Received 8 November 2005; accepted 1 May 2006; published online 6 July 2006)

\begin{abstract}
A select-divide-and-conquer variational method to approximate configuration interaction (CI) is presented. Given an orthonormal set made up of occupied orbitals (Hartree-Fock or similar) and suitable correlation orbitals (natural or localized orbitals), a large $N$-electron target space $S$ is split into subspaces $S_{0}, S_{1}, S_{2}, \ldots, S_{R}$. $S_{0}$, of dimension $d_{0}$, contains all configurations $K$ with attributes (energy contributions, etc.) above thresholds $\mathcal{T}_{0} \equiv\left\{T_{0}^{\text {egy }}, T_{0}^{\text {etc. }}\right\}$; the CI coefficients in $S_{0}$ remain always free to vary. $S_{1}$ accommodates $K$ s with attributes above $\mathcal{T}_{1} \leqslant \mathcal{T}_{0}$. An eigenproblem of dimension $d_{0}+d_{1}$ for $S_{0}+S_{1}$ is solved first, after which the last $d_{1}$ rows and columns are contracted into a single row and column, thus freezing the last $d_{1}$ CI coefficients hereinafter. The process is repeated with successive $S_{j}(j \geqslant 2)$ chosen so that corresponding CI matrices fit random access memory (RAM). Davidson's eigensolver is used $R$ times. The final energy eigenvalue (lowest or excited one) is always above the corresponding exact eigenvalue in $S$. Threshold values $\left\{\mathcal{I}_{j} ; j=0,1,2, \ldots, R\right\}$ regulate accuracy; for large-dimensional $S$, high accuracy requires $S_{0}+S_{1}$ to be solved outside RAM. From there on, however, usually a few Davidson iterations in RAM are needed for each step, so that Hamiltonian matrix-element evaluation becomes rate determining. One $\mu$ hartree accuracy is achieved for an eigenproblem of order $24 \times 10^{6}$, involving $1.2 \times 10^{12}$ nonzero matrix elements, and $8.4 \times 10^{9}$ Slater determinants. (C) 2006 American Institute of Physics.
\end{abstract} [DOI: $10.1063 / 1.2207621]$

\section{INTRODUCTION}

Quantum chemistry largely revolves around the development and application of methods to approximate Schrödinger's equation for stationary states,

$$
H \Psi_{\mu}=E_{\mu} \Psi_{\mu}
$$

Orbital basis set methods provide the most general approach ${ }^{1}$ when the number of active electrons is not too large. ${ }^{2}$ After selection of a suitable orbital set, which is essential to obtain meaningful chemical or physical results, ${ }^{3}$ the main question is what to do with a computationally intractable representation $\mathbf{H}$ of the Hamiltonian in the given orbital set,

$$
\mathbf{H C}_{\mu}=E_{\mu} \mathbf{C}_{\mu} .
$$

Naturally, this complete or full configuration interaction (CI) needs to be drastically simplified, resulting in several approximate methods. ${ }^{1,4-10}$ Within the CI approaches, highly correlated configuration interaction ${ }^{11}$ (HCCI) methods, viz., CI going well beyond the singles and doubles treatment, constitutes a practical alternative to using a full Hamiltonian representation, particularly after the developments in the companion paper ${ }^{12}$ to be referred to as I.

One possibility is to select a priori a subspace $S$ having invariant properties with respect to separate unitary transformations of the occupied orbitals and of distinct sets of cor-

\footnotetext{
${ }^{a)}$ Electronic mail: bunge@ fisica.unam.mx

${ }^{b)}$ Electronic mail: quantumqsar@hotmail.com
}

relation orbitals grouped in a well-defined manner, ${ }^{11}$ giving rise to multireference CI (MRCI) ${ }^{13-18}$ complete active space (CAS) $\mathrm{CI}^{19}$ restricted active space (RAS) $\mathrm{CI}^{20}$ and so on. ${ }^{21}$ The corresponding CI-matrix eigenvalue problem is

$$
\mathbf{H}^{S} \mathbf{C}=E_{S} \mathbf{C},
$$

where the subindex $\mu$ was dropped in the understanding that the following also applies to excited states. Equation (3) is often solved by Davidson's method. ${ }^{22,23}$ Typically, an accuracy of $10^{-8}$ a.u. for a ground state requires 40-60 iterations dominated by the evaluation of a vector $\sigma$,

$$
\sigma=\mathbf{H v},
$$

in terms of an approximate eigenvector $\mathbf{v}$. In a basis of $\mathrm{N}$-electron symmetry eigenfunctions or configuration-statefunctions (CSFs) the corresponding $H_{\mu \nu}$ s are given by lengthy formulas whose application may require hundreds of computer processor cycles, justifying to store them in disk memory after being evaluated only once. Thus in a CSF framework, the upper-limit size for evaluation of Eq. (4) is determined by the capacity of the disks employed and by the willingness to spend comparatively large amounts of time to retrieve $\mathbf{H}$ while the computer processors remain idle or are used by competing programs. Alternatively, for determinantal CI spaces, each nonzero $H_{\mu \nu}$ can be evaluated in a few cycles of computer time ${ }^{11}$ so that the entire Hamiltonian matrix can be recalculated on-the-fly as needed for each iteration of Eq. (4) therefore overcoming the necessity to store 
$H_{\mu \nu} \mathrm{S}$ in disk. This was, in fact, the reason behind the move from CSFs to determinants about two decades ago. ${ }^{20,24}$

Concurrently, and departing from invariant spaces, another possibility was advanced in the previous paper ${ }^{12}$ where it was shown that a model space $M$ can be split a priori into a selected space $S$ harboring the energetically most important configurations, and a remainder whose energy effect $\Delta E$ can be predicted with fair accuracy through variational-like estimates before embarking on an HCCI calculation. The corresponding energy $E_{M}$ is given by

$$
E_{M}=E_{S}+\Delta E+\delta E,
$$

where $\delta E$ is a residual error that can be estimated after some work. ${ }^{12}$ Whatever HCCI method is invoked, the energy of a stationary state can be approximated by the rhs of Eq. (5) which in turn demands the solution of (3). In order to obtain small and reliable $\Delta E$ and $\delta E$ values, however, it is still necessary to deal with $S$ spaces which are too large to handle by modern HCCI. ${ }^{11}$

If the input-output bottleneck of the eigenproblem ${ }^{25}$ is overcome, a return to CSFs has the following advantages: (i) the number of nonzero $H_{\mu \nu}$ s is significantly reduced, resulting in a corresponding reduction of the number of arithmetic operations to evaluate Eq. (4), (ii) by diminishing the size of vectors $\sigma$ and $\mathbf{v}$ by several orders of magnitude, data localization is improved thus speeding up data transit between memory hierarchies, and (iii) excited states are readily and unequivocally identified. While retaining Davidson's or similar algorithms, ${ }^{26}$ this paper presents a select-divide-andconquer variational method (SDC-CI) for the specific solution of the atomic or molecular CI-matrix eigenvalue problem with little or no resort to external storage devices. After introducing CI notation and a general strategy to select configurations in Sec. II, SDC-CI is formulated in Sec. III. An example and a general discussion are given in Sec. IV.

\section{MOTIVATION, CI NOTATION AND SELECTED Cl}

\section{A. Motivation}

Let $S$ be a target space calling for a CI treatment. It is convenient that $S$ be made up of CSFs. These are built up from a suitable orthonormal set of occupied orbitals. As before, $\mathbf{H}^{S}$ is the representation of the Hamiltonian in $S$;

$$
\mathbf{H}^{S} \mathbf{C}=E_{S} \mathbf{C} .
$$

Here we have rewritten Eq. (3) just to emphasize a new and necessary requirement [not present in Eq. (3)]. The use of natural orbitals ${ }^{27,28}$ or localized orbitals ${ }^{29}$ as correlation orbitals, in order to facilitate an a priori and quantitative selection and deletion of CSFs. ${ }^{12}$ Distinct from general eigenproblems, Eq. (6) is a very special eigenvalue problem in which various eigenvector components are related among themselves in a way which can be anticipated ${ }^{12}$ before knowing its solution. This circumstance allows the subdivision of $S$ into subspaces $S_{0}, S_{1}, S_{2}, \ldots, S_{R}$ each characterized by configurations with decreasing importance in their energy contributions. Other criteria to select $S_{i}$ subspaces will also be discussed.

\section{B. Cl notation and Brown's formula}

A general HCCI wave function can be written as ${ }^{30}$

$$
\Psi=\sum_{K=1}^{K_{x}} \sum_{g=1}^{g_{K}} F_{g K} C_{g K} .
$$

In Eq. (7), $K$ and $g$ label configurations and degenerate elements, respectively, and $C_{g K}$ denotes a CI coefficient. $F_{g K}$ is a CSF expressed as a linear combination of Slater determinants $D_{i K}$,

$$
F_{g K}=O(\Gamma, \gamma) \sum_{i=1}^{g} D_{i K} b_{i}^{g}=\sum_{i=1}^{n_{K}} D_{i K} c_{i}^{g},
$$

where $O(\Gamma, \gamma)$ is a symmetric projection operator ${ }^{31}$ for all appropriate symmetry operators $\Gamma$ and a given irreducible representation $\gamma \cdot{ }^{32}$ The calculations in this paper will use the full range of $g_{K}$ degenerate elements, although this is not necessary, in general. ${ }^{30,33}$

Given a CI wave function $\Psi$, the energy contribution $\Delta E_{g K}$ of $F_{g K}$ is defined as

$$
\Delta E_{g K}=\langle\Psi|H| \Psi\rangle-\left\langle\Psi\left(-F_{g K}\right)|H| \Psi\left(-F_{g K}\right)\right\rangle,
$$

where $\Psi\left(-F_{g K}\right)$ denotes $\mathcal{N}\left(\Psi-F_{g K} C_{g K}\right)$ and $\mathcal{N}$ is a normalization factor, such that the wave function $\Psi\left(-F_{g K}\right)$ has the same remaining expansion coefficients as $\Psi$ except for renormalization. $\Delta E_{g K}$ can be approximated by Brown's formula, $^{34}$

$$
\Delta E_{g K}=\left(E-H_{g K, g K}\right) C_{g K}^{2} /\left(1-C_{g K}^{2}\right),
$$

where $E=\langle\Psi|H| \Psi\rangle$. Equation (7) is now rewritten as

$$
\Psi=\sum_{K=1}^{K_{x}} G_{K} B_{K}
$$

in terms of normalized symmetry configurations $G_{K}$,

$$
\begin{aligned}
& G_{K}=\operatorname{Sign} \cdot N_{K} \sum_{g=1}^{g_{K}} F_{g K} C_{g K}, \\
& B_{K}=\frac{1}{N_{K}}, \quad N_{K}=\operatorname{Sign} \sqrt{1 / \sum_{g=1}^{g_{K}} C_{g K}^{2}},
\end{aligned}
$$

in an obvious notation. In Eq. (12) and (13), Sign is the minus sign if the contribution of negative $C_{g K}$ coefficients to the sum of squares is larger than the one provided by the positive coefficients, and vice versa. Unsigned $B_{K}$ coefficients are also of interest. ${ }^{12}$ Similarly as $\Delta E_{g K}$ in Eq. (10), $\Delta E_{K}$ for expansion (11) is given by

$$
\Delta E_{K}=\left(E-H_{K K}\right) B_{K}^{2} /\left(1-B_{K}^{2}\right),
$$

which is used just for estimating the truncation energy error. A thorough discussion of Eq. (14) together with predictive formulas for $B_{K}$ coefficients up to sextuply excited configurations is found in Paper I. 


\section{Energy threshold $T^{\text {egy }}$}

Brown's formula provides a useful criterion to select configurations $K$ based on energy thresholds $T^{\text {egy }}$, provided the coefficients $B_{K}$ can be predicted. This happens when $K$ can be formed as a product of combinations of singly and doubly excited configurations, such as any triply or quadruply excited configuration in a MRCI singles and doubles out of a single reference configuration. Such configurations are called disconnected configurations. ${ }^{12}$ A subdivision of $S$ into subspaces $S_{j},(j=0,1,2, \ldots, R)$ can be partially characterized by

$$
\begin{aligned}
& \left|\Delta E_{K}\right|>T_{j}^{\text {egy }}, \\
& T_{0}^{\text {egy }} \geqslant T_{1}^{\text {egy }} \geqslant T_{2}^{\text {egy }} \geqslant \ldots \geqslant T_{R}^{\text {egy }} .
\end{aligned}
$$

\section{Occupation number threshold $T^{\text {on }}$}

The rest of the configurations are called connected configurations. They are selected according to occupation number thresholds $T^{\text {on }}$ based on density matrix concepts. As already mentioned, the SDC-CI method may require the correlation orbitals $a, b, c$ to be approximate natural orbitals, ${ }^{27,28}$ viz., eigenfunctions $\chi_{a}$ of the reduced first-order density matrix $\gamma\left(1,1^{\prime}\right)$,

$$
\gamma\left(1,1^{\prime}\right)=\sum n_{a} \chi_{a}^{*}(1) \chi_{a}\left(1^{\prime}\right),
$$

where the $n_{a}$ s are the eigenvalues or occupation numbers. Alternatively, in extended molecules, if localized orbitals ${ }^{29}$ are used, the occupation numbers $n_{a}$ have to be replaced by effective occupation numbers $n_{a}^{\text {eff }}$,

$$
n_{a}^{\mathrm{eff}}=\sqrt{\sum_{j}\left|\gamma_{a j}\right|^{2}} \text {. }
$$

For each $q$-excited configuration $K$ the product $P(q, K)$ of corresponding occupation numbers is given by

$$
P(q, K)=\prod_{i=1}^{q} n_{K_{i}},
$$

where $K_{i}$ represents either a correlation natural orbital or a localized orbital. The characterization of the subspaces $S_{j}$, $(j=0,1,2, \ldots, R)$ is enriched by enforcing

$$
\begin{aligned}
& P(q, K)>T_{j}^{\text {on }} . \\
& T_{0}^{\text {on }} \geqslant T_{1}^{\text {on }} \geqslant T_{2}^{\text {on }} \geqslant \cdots \geqslant T_{R}^{\text {on }} .
\end{aligned}
$$

In (20) and (21), $T_{j}^{\text {on }}$ may be a number or, more generally, a suitable function of some parameters,

$$
T^{\text {on }}(m)=F_{\text {dh }} F_{\text {con }} 10^{-m g},
$$

where $F_{\mathrm{dh}} \geqslant 1$ is a deep-hole factor ${ }^{12}$ associated to holes involving inner electrons, and $F_{\text {con }} \geqslant 1$ is a factor for connected configurations. ${ }^{12}$ The introduction of $F_{\mathrm{dh}}$ and $F_{\text {con }}$ is to recognize the well characterized families of configurations that are comparatively less important for a given value of $P(q, K){ }^{12}$ The parameter $m$ is shown explicitly on the lefthand side (lhs) of (22) for later purposes. $10^{-m}\left(F_{\mathrm{dh}} F_{\text {con }}\right)^{1 / 4}$ may be interpreted as an average occupation number below which configurations involving that natural orbital are deleted from an original model space $M$.

\section{E. Harmonic truncation threshold $T^{\text {har }}$}

A final subdivision of $S$ into subspaces $S_{j}$, $(j=0,1,2, \ldots, R)$ is specified by a harmonic truncation threshold $T_{j}^{\text {har }}$ indicating that subspace $S_{j}$ is truncated in the orbital basis after a given harmonic $\ell_{j}$,

$$
T_{j}^{\mathrm{har}}=\frac{1}{\ell_{j}} .
$$

Resort to a fraction is dictated by the rule that first occurring subspaces have larger thresholds than subspaces further down the sequence

$$
T_{0}^{\text {har }} \geqslant T_{1}^{\text {har }} \geqslant T_{2}^{\text {har }} \geqslant \cdots \geqslant T_{R}^{\text {har }} .
$$

\section{F. Selection strategy}

A subspace $S_{j}$ consists of the following configurations:

(i) All (connected and disconnected) triples with $T_{j-1}^{\text {on }}$ $\geqslant P(3, K) \geqslant T_{j}^{\text {on }}$. Here, the occurrence of disconnected triples is due to their carrying connected contributions in the many-body perturbation theory sense. $^{12}$

(ii) Of the remaining (disconnected) triples, those with $T_{j-1}^{\text {egy }} \geqslant\left|\Delta E_{K}\right|>T_{j}^{\text {egy }}$.

(iii) Connected quadruples with $T_{j-1}^{\text {on }} \geqslant P(4, K)>T_{j}^{\text {on }}$.

(iv) Disconnected quadruples with $T_{j-1}^{\text {egy }} \geqslant\left|\Delta E_{K}\right|>T_{j}^{\text {egy }}$. Here it is assumed that disconnected quadruples have negligible connected contributions; otherwise, they must also enter in (iii).

(v) Quintuple- and higher-excited configurations can be selected according to (iii) and (iv).

(vi) Finally, for atoms, $S_{j}$ contains configurations with harmonics up to $1 / T_{j}^{\text {har }}$. A similar threshold for molecules may be provided if warranted.

\section{SELECT-DIVIDE-AND-CONQUER CI}

In synthesis, the target space $S$ is split into subspaces $S_{0}, S_{1}, S_{2}, \ldots, S_{R}$ each characterized by configurations $\left\{\Phi_{i j}\right.$; $\left.i=1,2, \ldots, d_{j} ; \quad j=0,1, \ldots, R\right\} \quad$ above thresholds $\left\{\mathcal{T}_{j}\right.$ $\left.\equiv T_{j}^{\text {egy }}, T_{j}^{\text {on }}, T_{j}^{\text {har }} ; j=0,1, \ldots, R\right\}$ :

$$
\begin{aligned}
& S_{0} \equiv\left\{\Phi_{i 0} ; i=1,2, \ldots, d_{0}\right\}, \quad T_{0}^{\text {egy }}, T_{0}^{\text {on }}, T_{0}^{\text {har }}, \\
& S_{1} \equiv\left\{\Phi_{i 1} ; i=1,2, \ldots, d_{1}\right\}, \quad T_{1}^{\text {egy }}, T_{1}^{\text {on }}, T_{1}^{\text {har }}, \\
& S_{j} \equiv\left\{\Phi_{i j} ; i=1,2, \ldots, d_{j}\right\}, \quad T_{j}^{\mathrm{egy}}, T_{j}^{\mathrm{on}}, T_{j}^{\mathrm{har}},
\end{aligned}
$$

with

$$
\begin{aligned}
& T_{0}^{\text {egy }} \geqslant T_{1}^{\text {egy }} \geqslant T_{j}^{\text {egy }}, \\
& T_{0}^{\text {on }} \geqslant T_{1}^{\text {on }} \geqslant T_{j}^{\text {on }},
\end{aligned}
$$




$$
T_{0}^{\mathrm{har}} \geqslant T_{1}^{\mathrm{har}} \geqslant T_{j}^{\mathrm{har}}
$$

Successive $S_{j}(j \geqslant 2)$ are chosen so that corresponding CI matrices fit random access memory (RAM). We start by solving an eigenproblem of dimension $d_{0}+d_{1}$ in $S_{0}+S_{1}$,

$$
\mathbf{H}^{S_{0}+S_{1}} \mathbf{C}^{(0+1)}=E^{(0+1)} \mathbf{C}^{(0+1)},
$$

after which the last $d_{1}$ rows and columns are contracted into a single row and column,

$$
\begin{aligned}
& \mathbf{H}_{i, d_{0}+1}=\sum_{j=1}^{d_{1}}\left\langle\Phi_{i 0}|H| \Phi_{j 1}\right\rangle C_{i 0} C_{j 1}, \\
& \mathbf{H}_{d_{0^{+1}, d_{0}+1}}=\sum_{j=1}^{d_{1}} \sum_{j^{\prime}=1}^{d_{1}}\left\langle\Phi_{j 1}|H| \Phi_{j^{\prime} 1}\right\rangle C_{j 1} C_{j^{\prime} 1},
\end{aligned}
$$

giving

$$
\mathbf{H}^{S_{0}+c_{1}} \mathbf{C}^{\left(0+c_{1}\right)}=E^{\left(0+c_{1}\right)} \mathbf{C}^{\left(0+c_{1}\right)},
$$

where subspace $S_{1}$ was contracted into a single dimensional space $c_{1}$; of course, $E^{(0+1)}$ in (27) is equal to $E^{\left(0+c_{1}\right)}$ in (30) except for roundoff errors. This amounts to freeze the last $d_{1}$ CI coefficients hereinafter. Upon completion of this first step, the resulting matrix of dimension $d_{0}+1$ is enlarged by adding $d_{2}$ rows and columns associated to $S_{2}$,

$$
\mathbf{H}^{S_{0}+c_{1}+S_{2}} \mathbf{C}^{\left(0+c_{1}+2\right)}=E^{\left(0+c_{1}+2\right)} \mathbf{C}^{\left(0+c_{1}+2\right)},
$$

and the process is repeated,

$$
\begin{aligned}
& \mathbf{H}^{S_{0}+s_{r}+S_{r}} \mathbf{C}^{\left(0+s_{r}+r\right)}=E^{\left(0+s_{r}+r\right)} \mathbf{C}^{\left(0+s_{r}+r\right),} \\
& s_{r}=\sum_{q=1}^{r-1} c_{q},
\end{aligned}
$$

until incorporation of subspace $S_{R}$ exhausts the target space $S$. Each step yields increasingly accurate eigenvalues $E^{\left(0+s_{r}+r\right)}$ converging from above, and wave functions $\Psi^{(r)}$,

$$
\begin{aligned}
& \Psi^{(r)}=\sum_{i=1}^{d_{0}} \Phi_{i 0} C_{i 0}^{(r)}+\sum_{q=1}^{r-1} \Phi^{(q)} C_{q}^{(r)}+\sum_{j=1}^{d_{r}} \Phi_{j r} C_{j r}, \\
& (r=1,2, \ldots, R), \\
& \Phi^{(q)}=N_{q} \sum_{i=1}^{d_{q}} \Phi_{i q} C_{i q}, \quad(q=1,2, \ldots, R) .
\end{aligned}
$$

In practice, the value of $R$ may attain several thousands. In the present computer code, $d_{0}+r-1+d_{r}$ is required not to exceed 65536 , so that each index $\mu$ and $\nu$ in $H_{\mu \nu}$ can be stored in only two bytes (16 bits), a demand that can be lifted for large RAM memories. (We actually use suitable offsets to extend the range of $\mu$ and $\nu$ values to a large extent past 65536 while still using two bytes per index.)

Since $H_{\mu \nu} \mathrm{S}$ with $\mu \leqslant d_{0}, \nu \leqslant d_{0}$, are present in all CI matrices, the CI coefficients $C_{i 0}^{(r)}$ in $S_{0}$ always remain free to vary. Clearly, the final energy eigenvalue $E^{\left(0+s_{R}+R\right)}$ (lowest or an excited one) is at or above its exact partner in $S$,
TABLE I. Convergence of the Ne ground state energy with $T_{1}^{\text {on }}$ and $T_{1}^{\text {har }}$; $T_{1}^{\text {egy }}=10^{-11}$ a.u. is kept fixed.

\begin{tabular}{lccrr}
\hline \hline$m$, Eq. (21) & $\ell$ & $d_{0}+d_{1}$ & $-E^{(0+1)}$ & $-E^{\left(0+s_{R}+R\right)}$ \\
\hline 4 & 3 & 265189 & 128.93523456 & 128.93651267 \\
4.5 & 4 & 440159 & 128.93600860 & 128.93651601 \\
5 & 4 & 559235 & 128.93601535 & 128.93661665 \\
5.5 & 4 & 779431 & 128.93601746 & 128.93651685 \\
6 & 4 & 1080552 & 128.93601794 & 128.93651699 \\
4.5 & 5 & 550097 & 128.93624019 & 128.93651533 \\
5 & 5 & 818263 & 128.93625066 & 128.93651631 \\
4.5 & 6 & 622138 & 128.93632365 & 128.93651492 \\
5 & 6 & 1055538 & 128.93633641 & 128.93651615 \\
\hline \hline
\end{tabular}

$$
E_{S}=E^{\left(0+s_{R}+R\right)}-\delta \epsilon,
$$

where $\delta \epsilon$ is another residual error (with positive sign) that needs to be assessed. The first two terms of the sequence $\left\{\mathcal{T}_{j}\right\}, \mathcal{T}_{0}$, and $\mathcal{T}_{1}$, are the main threshold values regulating $\delta \epsilon$, as illustrated in the next section.

\section{APPLICATION AND DISCUSSION}

\section{A. Application and accuracy}

SDC-CI interferes with the rigorous solution of the eigenproblem (6) by freezing most of the linear variational coefficients after a first variational estimate on a relatively small subspace of CSFs. Only the linear variational coefficients of CSFs in $S_{0}$ are free to vary all the time. Both the energy and the wave function are affected by these variational constraints; we shall only consider energy effects, exhibited by $\delta \epsilon$ in Eq. (34).

As an example, we choose the CI matrix of the $\mathrm{Ne}$ ground state for one of the largest calculations of Paper I. The model space $M$ is a full CISDTQ (CI singles, doubles, triples, and quadruples) in a $12 s 12 p 11 d 10 f 10 g 9 h 8 i 7 k 6 l 5 m 4 n 3 o 3 q 3 r$ orbital basis spanning a CSF space of dimension $1.4 \times 10^{9}$, and involving $1.1 \times 10^{12}$ distinct Slater determinants. ${ }^{12}$ This model space $M$ is pruned before evaluation of symmetry eigenfunctions, as discussed in Paper I; the pruned subspace $P$ is about one sixth the size of $M$, and carries a truncation energy error about $13 \mu$ hartree (Ref. 12) with respect to $M$, which is mentioned just to clarify the general context.

The target subspace $S$ is obtained by truncating the pruned subspace $P$ with the following thresholds: $T_{R}^{\text {egy }}$ $=10^{-11}$ a.u., and $T_{R}^{\text {on }}$ given by Eq. (22) with $m=6.5\left(T^{\text {on }}\right.$ $\left.=F_{\mathrm{dh}} F_{\mathrm{con}} \cdot 3 \times 10^{-7}\right)$. Thus, $S$ is here obtained by the selected CI (SCI) method. ${ }^{12}$ As it turns out, subspace $S$ harbours 859903 configurations, $24.06 \times 10^{6} \mathrm{CSFs}, 8.36 \times 10^{9}$ detors, and gives rise to $1.18 \times 10^{12}$ nonzero Hamiltonian matrix elements. The truncation energy error accumulated in going from the pruned space $P$ into the selected space $S$ is of no concern here but is carefully discussed in Paper I.

We now focus on assessing the uncertainty $\delta \epsilon$ in Eq. (34). In Table I the SDC-CI energy $E^{\left(0+s_{R}+R\right)}$ (fifth column) is shown as a function of $T_{1}^{\text {on }}$ values (first column), and also as a function of the highest $\ell$ value in the orbitals spanning the $S_{0}$ and $S_{1}$ subspaces (second column). The remaining perti- 
nent thresholds are fixed $T_{0}^{\text {egy }}=10^{-5}$ a.u., $T_{1}^{\text {egy }}=10^{-8}$ a.u., and $T_{j}^{\text {egy }}=10^{-11}$ a.u. $(j=2,3, \ldots, R)$; the $T_{j}^{\text {on }} \mathrm{s}$ are given by Eq. (21) with $m=2.7(j=0)$, and $m=6.5(j=2,3, \ldots, R)$, respectively. The third and fourth columns show the order $d_{0}$ $+d_{1}$ of the matrix to be treated outside RAM, and the corresponding energy eigenvalue, respectively. It is seen that achieving a low energy eigenvalue $E^{0+1}$ in $S_{0}+S_{1}$ is not a requisite for an accurate energy $E^{\left(0+s_{R}+R\right)}$.

As thresholds are tightened, the evolution of the energy $E^{\left(0+s_{R}+R\right)}$ towards the exact result $E_{S}$ (usually unknown and unattainable with contemporary workstations), is from above, of course. Monotonic behavior is generally obtained for fixed values of $T_{0}^{\text {har }}$ and $T_{1}^{\text {har }}$. The most favorable results, viz., those with lowest and most rapidly convergent energies, occur for $T^{\text {har }}=1 / \ell=1 / 4$, and are used to push down $T^{\text {on }}$ hoping to reach adequate convergence, as shown in lines 2-5 of Table I. These results suggest an accuracy better than $1 \mu$ hartree $(\delta \epsilon=1 \mu$ hartree), which is also supported by smooth energy convergence patterns in Paper I, at the onetenth of $1 \mu$ hartree level. This is more precise than the few $\mu$ hartree typically used to assess convergence in full $\mathrm{CI}$ benchmarks. ${ }^{35}$

The occurence of energy oscillations with decreasing values of $T^{\text {har }}$ shows that significant interactions are being frozen at various steps, thus imposing constraints which impair the full extent of the variational procedure to the degree shown in the last column of Table I. The problem is finding an appropriate way to select $a$ priori those sets of configurations that must be taken together in order to achieve a desired accuracy. Our approach here has been circumscribed to vary $T_{1}^{\text {on }}$ and $T_{1}^{\text {har }}$ thresholds after reaching convergence with the less sensitive thresholds $T_{1}^{\text {egy }}$ and $\mathcal{T}_{0}$. Another obvious criterion (not considered) is to limit natural orbitals in $S_{1}$ to those with occupation numbers above some threshold. At any rate, there is still much to be learnt about accuracy control in SCI-CI.

The studies of Table I were repeated by changing the occupied orbitals from Hartree-Fock to Brueckner ones, ${ }^{36,37}$ viz., orbitals for which the coefficients of singly excited configurations are zero. Brueckner orbitals were approximated in the framework of a CISD using a general method, ${ }^{38}$ valid for the ground state, and also for excited states. Unfortunately, the results were similar to those shown in Table I, the energy converging $24 \mu$ hartree above the one obtained with the Hartree-Fock orbitals, indicating a higher energy limit for CISDTQ of Ne ground state when calculated in terms of Brueckner orbitals instead of the Hartree-Fock orbitals.

\section{B. Further thoughts}

Since Davidson's eigensolver is used $R$ times, the usual threshold for energy convergence in Davidson's algorithm must be divided by $R$, viz., for an accuracy of $10^{-8}$ a.u. and $R=1000$, each Davidson application must be required to converge to within $10^{-11}$ a.u.

High accuracy may require a large value of $d_{0}+d_{1}$ demanding the eigenproblem in $S_{0}+S_{1}$ to be solved outside RAM. That, however, only affects the first iteration. From there on, all work proceeds in RAM. Also, in general, just a few Davidson iterations are needed for each step, even with such tight energy convergence threshold as $10^{-11}$ a.u. for each Davidson application, since the most important energy contributions and eigenvector components are already in $S_{0}$ and $S_{1}$. Moreover, because of the relatively small dimension of the vectors $\sigma$ and $\mathbf{v}$ in (4), the data set is considerably more localized. Profiles of program execution running $13 \mathrm{~h}$ entirely in RAM (Ref. 12) show that about $8 \%$ of the time is spent on Eq. (4) (3\% on code in RAM and $5 \%$ on code using disk), the rest being used mainly in the evaluation of $H_{\mu \nu}$ s.

\section{Comparison with previous work and outlook}

The idea to freeze CI coefficients is not a new one. Back in 1969, one of the authors (Bunge) was asked ${ }^{39}$ whether it would be sensible to use the configurational expansion Eq. (7) in small chuncks, then lock $C_{g K}$ coefficients $\left(g=1,2, \ldots, g_{K}\right)$ by Eq. (12), and finally use the corresponding configurations in the less general Eq. (11). Formally, after some generalization, the idea can be embodied in Eqs. (27)-(33). In retrospective, had Schaefer's suggestion been seriously pursued, the ingredients of SCI to achieve acceptable accuracy might have appeared shortly afterwards, probably following Kutzlenigg's review on electron-pair theories ${ }^{40}$ and Shavitt's review on CI (Ref. 41) in consecutive chapters of Schaefer's volume 3 of Modern Theoretical Chemistry. More recently, the other author (Carbó-Dorca) played with the idea of avoiding the eigenproblem altogether; ${ }^{42}$ that idea was also short of providing the necessary accuracy. These two ideas, however, met with SCI needs and SCI possibilities, giving way to SDC-CI.

In an effort to lower the size of MRCI expansions, internally contracted MRCI was proposed. ${ }^{43}$ Meyer's and similar approaches ${ }^{11}$ using perturbation theory estimates of various CI coefficients are the nearest relatives of SDC-CI. The latter is more accurate at the expense of having to evaluate all pertinent matrix elements. We encourage young workers to apply perturbation theory ${ }^{44,45}$ to SDC-CI in an effort to search for a middle ground between our's and Meyer's ideas, where not all matrix elements would be necessary.

Other investigations are also warranted: (i) to reduce $\delta \epsilon$ in Eq. (34), (ii) to lessen the computational time to evaluate $H_{\mu \nu} \mathrm{s}$, and (iii) to run simultaneously on many processors. With regard to (i), more flexible selections of subspaces have to be examined. Wholesale evaluation of $H_{\mu \nu}$ s, on the other hand, is open to several theoretical alternatives: for atoms, ${ }^{33,46}$ and for molecules, ${ }^{11,33}$ and a wealth of computer implementations yet to be explored.

\section{Conclusions}

Typical $\mathbf{H}^{S}$ matrices encountered in SCI need tens or hundreds of terabytes of disk to store expensive-to-evaluate $H_{\mu \nu} \mathrm{s}$, and considerable disk retrieval performance for each Davidson iteration. ${ }^{47,48}$ A general eigensolver has to face the full implications of such disk-read bottleneck, ${ }^{25,48}$ and pressing demands through memory hierarchies to access widely scattered elements of the vectors $\sigma$ and $\mathbf{v}$ in the evaluation of (4). The computationally tractable SDC-CI method, on the other hand, overcomes the input-output bottleneck and part 
of the scattered data problem, and can be applied to a target space $S$ provided the orbital set is made up of Hartree-Fock (or Brueckner) occupied orbitals, plus approximate natural orbitals (or localized orbitals). The price paid by SDC-CI is a small loss of accuracy relative to general algorithms ${ }^{22,23}$ when both methods can be used. This small loss of accuracy, however, is more than compensated by the SDC-CI capability to deal with CI matrices having trillions of expensive-toevaluate nonzero matrix elements between $\mathrm{CSFs}^{12}$ much larger than ever attempted by other methods on a single processor, and comparable with a recent calculation on 432 processors. $^{48}$

SCI (Ref. 12) and SDC-CI are intimately intertwined: without SDC-CI the accuracy of SCI would fall short by several orders of magnitude, and without SCI, SDC-CI cannot even be formulated. For this reason, we believe that all HCCI methods eventually making use of SDC-CI will ultimately be assimilated into an SCI framework. The reverse, however, is not true, selection of configurations finds application in many other methods that do not require a variational solution. ${ }^{12}$

${ }^{1}$ I. Lindgren and J. Morrison, Atomic Many-Body Theory (Springer-Verlag, Berlin, 1986).

${ }^{2}$ W. Kohn, Rev. Mod. Phys. 71, 1253 (1999).

${ }^{3}$ H. F. Schaefer III, The Electronic Structure of Atoms and Molecules (Addison Wesley, Reading, MA, 1972).

${ }^{4}$ J. Linderberg and Y. Öhrn, Propagators in Quantum Chemistry (Academic, New York, 1973).

${ }^{5}$ F. E. Harris, H. J. Monkhorst, and D. L. Freeman, Algebraic and Diagrammatic Methods in Many-Fermion Theory (Oxford University Press, New York, 1992).

${ }^{6}$ M. Nakata, H. Nakatsuji, M. Ehara, M. Fukuda, K. Nakata, and K. Fujisawa, J. Chem. Phys. 114, 8282 (2001).

${ }^{7}$ S. R. White and R. L. Martin, J. Chem. Phys. 110, 4127 (1999).

${ }^{8}$ H. Nakatsuji, J. Chem. Phys. 113, 2949 (2000).

${ }^{9}$ H. Nakatsuji and E. R. Davidson, J. Chem. Phys. 115, 2000 (2001).

${ }^{10}$ D. A. Mazziotti, J. Chem. Phys. 121, 10957 (2004).

${ }^{11}$ C. D. Sherrill and H. F. Schaefer III, in Advances in Quantum Chemistry, edited by P.-O. Löwdin (Academic, New York, 1999), Vol. 34, p. 143

${ }^{12}$ C. F. Bunge, J. Chem. Phys. 124, 014107 (2006), preceding paper.

${ }^{13}$ J. L. Whitten and M. Hackmeyer, J. Chem. Phys. 51, 5584 (1969).

${ }^{14}$ M. Hackmeyer and J. L. Whitten, J. Chem. Phys. 54, 3739 (1971).

${ }^{15}$ L. R. Kahn, P. J. Hay, and I. Shavitt, J. Chem. Phys. 61, 3530 (1974).

${ }^{16}$ R. J. Buenker and S. D. Peyerimhoff, Theor. Chim. Acta 35, 33 (1974).
${ }^{17}$ H.-J. Werner, in Advances in Chemical Physics, edited by K. P. Lawley (Wiley, New York, 1987), Vol. 69, pp. 1-62.

${ }^{18}$ R. Shepard, in Advances in Chemical Physics, edited by K. P. Lawley (Wiley, New York, 1987), Vol. 69, pp. 63-200.

${ }^{19}$ B. O. Roos, in Advances in Chemical Physics, edited by K. P. Lawley (Wiley, New York, 1987), pp. 399-445.

${ }^{20}$ J. Olsen, B. O. Roos, P. Jorgensen, and H. J. A. Jensen, J. Chem. Phys. 89, 2185 (1988).

${ }^{21}$ M. W. Schmidt and M. S. Gordon, Annu. Rev. Phys. Chem. 49, 233 (1998).

${ }^{22}$ E. R. Davidson, J. Comput. Phys. 15, 87 (1975).

${ }^{23}$ E. R. Davidson, Comput. Phys. 7, 519 (1994).

${ }^{24}$ P. J. Knowles and N. C. Handy, Chem. Phys. Lett. 111, 315 (1984).

${ }^{25}$ R. Shepard, I. Shavitt, and H. Lischka, J. Comput. Chem. 23, 1121 (2002).

${ }^{26}$ J. Bofill, J. Comput. Chem. 13, 101 (1999).

${ }^{27}$ P.-O. Löwdin, Phys. Rev. 97, 1474 (1955).

${ }^{28}$ P.-O. Löwdin and H. Shull, Phys. Rev. 101, 1730 (1956).

${ }^{29}$ C. Angeli, C. J. Calzada, R. Cimiraglia, S. Evangelisti, V. Gvihery, T. Leininger, J.-P. Malriev, D. Maynau, J. V. Pitarch Ruiz, and M. Sparta, Mol. Phys. 101, 1389 (2003).

${ }^{30}$ A. Bunge, J. Chem. Phys. 53, 20 (1970).

${ }^{31}$ E. P. Wigner, Group Theory (Academic, New York, 1959).

${ }^{32}$ A. V. Bunge, C. F. Bunge, R. Jáuregui, and G. Cisneros, Comput. Chem. (Oxford) 13, 201 (1989).

${ }^{33}$ O. Jitrik and C. F. Bunge, Phys. Rev. A 56, 2614 (1997).

${ }^{34}$ R. E. Brown, Ph.D. thesis, Department of Chemistry, Indiana University, 1967.

${ }^{35}$ E. Rossi, G. L. Bendazzoli, S. Evangelisti, and D. Maynau, Chem. Phys. Lett. 310, 530 (1999).

${ }^{36}$ R. K. Nesbet, Phys. Rev. 109, 632 (1958).

${ }^{37}$ P.-O. Löwdin, J. Math. Phys. 3, 1171 (1962).

${ }^{38}$ C. F. Bunge, R. Jáuregui, and E. Ley-Koo, Can. J. Phys. 76, 421 (1998).

${ }^{39}$ H. F. Schaefer III (personal communication).

${ }^{40}$ W. Kutzelnigg, in Modern Theoretical Chemistry, edited by H. F. Schaefer III (Plenum, New York, 1977), Vol. 3, p. 129.

${ }^{41}$ I. Shavitt, in Modern Theoretical Chemistry, edited by H. F. Schaefer III (Plenum, New York, 1977), Vol. 3, p. 189.

${ }^{42}$ E. Besalú and R. Carbó-Dorca, J. Math. Chem. 10, 100 (1998).

${ }^{43}$ W. Meyer, in Modern Theoretical Chemistry, edited by H. F. Schaefer III (Plenum, New York, 1977), Vol. 3, pp. 413-446.

${ }^{44}$ P. Claverie, S. Diner, and J.-P. Malrieu, Int. J. Quantum Chem. 1, 751 (1967).

${ }^{45}$ Z. Gershgorn and I. Shavitt, Int. J. Quantum Chem. 2, 751 (1968).

${ }^{46}$ Z. Rudzikas, Theoretical Atomic Spectroscopy (Cambridge University Press, Cambridge, 1997).

${ }^{47}$ P. Stampfuß and W. Wenzel, J. Chem. Phys. 122, 024110 (2005).

${ }^{48}$ Z. Gan and R. J. Harrison, presented at Supercomputing 2005, November 2005 (unpublished); http://sc05.supercomputing.org 